\title{
Non-familial Idiopathic Short Stature
}

National Cancer Institute

\section{Source}

National Cancer Institute. Non-familial Idiopathic Short Stature. NCI Thesaurus. Code C118690.

Idiopathic short stature in a child when neither parent is short in stature. 